\title{
For reprint orders, please contact: reprints@futuremedicine.com \\ Public awareness of heart attack symptoms: what should we look for and how will it help?
}

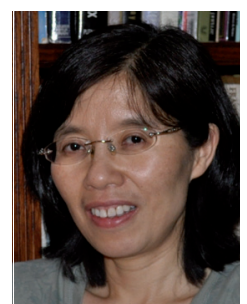

\author{
"Even with advanced reperfusion therapy for \\ heart attacks, the overall outcome would not \\ improve if patients do not arrive at a hospital early \\ enough to be eligible for the treatment."
}

Jing Fang

Division for Heart Disease \& Stroke Prevention, National Center for Chronic Disease Prevention \& Health Promotion, Centers for Disease Control \& Prevention, 4770 Buford Highway, NE, MS K-47, Atlanta, GA 30341-3717, USA = Tel..: +1 7704885142 - Fax: +1 7704888151 = jfang@cdc.gov

Today heart attacks are still one of the most common and deadly emergencies in the USA; each year there are approximately 610,000 new heart attacks and 325,000 recurrent attacks [1]. While new treatments such as thrombolytic drugs are available today, the efficiency of these medicines is ideal if they are administered within $1 \mathrm{~h}$ of symptom onset [2]. Reducing the length of time from the onset of the symptoms of heart attack to hospital arrival can increase the opportunity to use these therapies effectively in the early course of disease and can therefore increase the chance of survival.

Although sudden, dramatic 'Hollywood' heart attacks do occur, more often a heart attack begins with mild pain or pressure in the chest, which could extend through the shoulders, arms, back, teeth and jaw. Other symptoms may include lightheadedness, fainting, shortness of breath, nausea vomiting and fatigue. The symptoms of a heart attack may vary and may be similar to symptoms of other diseases, consequently a person may think that they have diesases other than heart attack and, therefore, wait too long before calling 911 or going to the hospital. Moreover, even individuals who are aware they are experiencing a heart attack may delay seeking care owing to a number of factors including fear, concerns about cost, self-treatment with medication, distrust of the healthcare system, consulting with family members, and embarrassment about calling emergency medical services if the condition turned out to not be a heart attack. Therefore, it is not surprising that a majority of those who experienced a heart attack had a delay of at least $2 \mathrm{~h}$ before they arrived at an emergency care setting, especially for those with lower socioeconomic status, such as women and minority ethnic groups [3].
This delay can be fatal. In the event of heart attack, reduced or stopped coronary blood flow leads to myocardial ischemia. If it is persistent, myocardial ischemia will result in tissue necrosis. Thrombolytic drugs or primary coronary angioplasty can reopen the blocked arteries and restore coronary blood flow. However, to be effective, these treatments should be administrated before tissue necrosis occurs.

\section{"Timely treatment is the key factor to reduce mortality from heart attack."}

Patients going to emergency care by car not only take longer to get to an emergency room, they may also miss the opportunity for artery cleaning procedures they would otherwise receive if they called 911. Currently, ambulance services are not only for transportation of the patient to an emergency care setting, they are also provided with equipment for diagnosis and immediate treatment, if needed. ECG monitors can detect a myocardial infarction and the ECG results can be sent to the hospital before the patient arrives. If the patient develops cardiac arrest, ambulance services can deliver immediate shocks to recover them from the arrest [4].

There have been various population-based activities to improve the awareness of heart attack symptoms and the importance of calling 911. 'Act in Time' campaign was launched by the American Heart Association and the National Heart, Lung and Blood Institute in 2001 [5,101]. Act in Time was being relaunched in 2010, though not as a media campaign, but rather as educational materials for consumers and providers. In addition, the US Centers for Disease Control and Prevention funds National Heart Disease and Stroke Prevention State

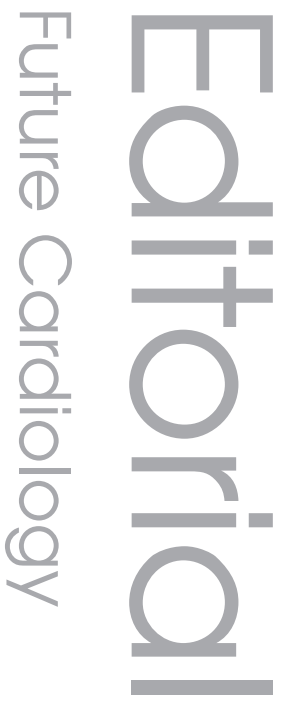

(1) 
Programs, increasing public awareness of heart attack symptoms is one of the objectives of the program [6,102].

However, national surveillance data show that the awareness of heart attack symptoms is still low among US adults - only $31 \%$ of US adults knew all five symptoms of a heart attack in 2005 [5], and this percentage did not improve when compared with 2001. Furthermore, although the benefits of reperfusion therapy were well accepted in recent decades, the time elapsed until hospital presentation among patients with acute myocardial infarction has not changed [6]. A recent report among women showed that in 2009, 54\% respondents indicated that heart disease was the leading cause of death among women in comparison with $30 \%$ in 1997; the percentage of awareness increased more among black women than white women. However, there was no noticeable improvement in the awareness of heart attack symptoms from 1997 to 2009. The symptom with the highest awareness (56\%) was chest pain and neck, shoulder and arm pain. The percentages of awareness of other symptoms were much lower, ranging from $29 \%$ for shortness of breath to $7 \%$ fatigue [7].

"Despite advances in effective treatment
for heart attack over the past decades,
improvements in decreasing the length of
time from onset of heart attack symptoms
to medical treatment have not
been observed."

Even with advanced reperfusion therapy for heart attacks, the overall outcome would not improve if patients do not arrive at a hospital early enough to be eligible for the treatment. So what should we do to improve public awareness of heart attack symptoms and the importance of receiving emergency care as soon as possible?

First, would it be more cost effective if the targeted educational campaigns were 'risk based'? The populations with the highest risk of cardiovascular disease are those who would benefit most through educational campaigns. These populations include patients with hypertension, high cholesterol, diabetes and atherosclerosis, as well as those who smoke cigarettes. Moreover, patients who survived previous heart attacks would have a higher risk of another event. These high-risk populations compose a large portion of the US adult population: in 2010, 17.6 million Americans have coronary heart disease, 35.7 million adults have cholesterol of $240 \mathrm{mg} /$ $\mathrm{dl}$ or greater, one in three adults have hypertension and $9.6 \%$ of adults from the US have diabetes [1]. In addition, the family members of patients with previous heart attack are also included in the high-risk group. Those individuals, for their own health interest, should be more willing to learn the signs and symptoms of heart attack.

\footnotetext{
${ }^{66}$ To reduce the delay time from the onset of symptoms to hospital arrival, public health efforts to improve awareness of heart attack symptoms may not be adequate without increased access to emergency care systems."
}

Second, while the cost of ambulance transportation varies depending on the location, it can be as high as US\$1200 per transport [103]. A recent study to examine the association between lack of health insurance, as well as underinsurance and the time from symptom onset to hospital presentation during heart attack revealed that $49 \%$ of uninsured, $45 \%$ of underinsured and $39 \%$ of insured individuals delayed care for greater than $6 \mathrm{~h}$ despite having symptoms of a heart attack [8], illustrating that efforts to educate the public about heart attack symptoms and calling 911 have limited effect if health insurance coverage is lacking or insufficient. Furthermore, the cost issue would have more impact on disadvantaged populations, including women and minorities since they are more likely to be uninsured or have financial concerns about medical costs, even for those who have insurance coverage. This study suggested that efforts to reduce prehospital delays for heart attacks may have limited benefits unless emergency care insurance coverage is extended and the affordability of care among the underinsured or those without insurance is improved.

Timely treatment is the key factor to reduce mortality from heart attack. The awareness of symptoms by the patient or bystanders is the most important factor to get timely treatment. Despite advances in effective treatment for heart attack over the past decades, improvements in decreasing the length of time from onset of heart attack symptoms to medical treatment have not been observed [2,3]. To reduce the delay time from the onset of symptoms to hospital arrival, public health efforts to improve awareness of heart attack symptoms may not be adequate without increased access to emergency care systems. 


\section{Financial \& competing interests disclosure}

The conclusions in this article are those of the author and do not represent the views of the Centers for Disease Control and Prevention. The author has no relevant affiliations or financial involvement with any organization or entity with a financial interest in or financial

\section{Bibliography}

1. Lloyd-Jones D, Adams RJ, Brown TM et al. Heart disease and stroke statistics - 2010 update: a report from the American Heart Association. Circulation 121, E46-E215 (2010).

2. Avorn J, Knight E, Ganz DA et al. Therapeutic delay and reduced functional status six months after thrombolysis for acute myocardial infarction. Am. J. Cardiol. 94, 415-420 (2004).

3. Foraker RE, Rose KM, McGinn AP. Neighborhood income, health insurance, and prehospital delay for myocardial infarction: the atherosclerosis risk in communities study. Arch. Intern. Med. 168(17), 1874-1879 (2008).

4. Hutchison AW, Malaiapan Y, Jarvie I et al. Prehospital 12-lead ECG to triage ST-elevation myocardial infarction and emergency department activation of conflict with the subject matter or materials discussed in the manuscript. This includes employment, consultancies, honoraria, stock ownership or options, expert testimony, grants or patents received or pending, or royalties.

No writing assistance was utilized in the production of this manuscript. the infarct team significantly improves door-to-balloon times: ambulance Victoria and MonashHEART Acute Myocardial Infarction (MonAMI) 12-lead ECG project. Circ. Cardiovasc. Interv. 2, 528-534 (2009).

5. Centers for Disease Control and Prevention. Disparities in adult awareness of heart attack warning signs and symptoms - 14 states, 2005. MMWR Morb. Mortal. Wkly Rep. 57, 175-179 (2008).

6. Goldberg RJ, Steg PG, Sadiq I et al. Extent of, and factors associated with, delay to hospital presentation in patients with acute coronary disease (the GRACE registry). Am. J. Cardiol. 89, 791-796 (2002).

7. Mosca L, Mochari-Greenberger H, Dolor RJ et al. Twelve-year follow-up of American women's awareness of cardiovascular disease risk and barriers to heart health. Circulation Cardiovasc. Qual. Outcomes 3, 120-127 (2010).
8. Smolderen KG, Spertus JA, Nallamothu BK et al. Health care insurance, financial concerns in accessing care, and delays to hospital presentation in acute myocardial infarction. JAMA 303, 1392-1400 (2010).

\section{Websites}

101. NHLBI. Acting in time to heart attack signs www.nhlbi.nih.gov/actintime/

102. CDC State Heart Disease and Stroke Prevention Program www.cdc.gov/dhdsp/state_program/index. htm

103. US Government Accountability Office. Report to congressional committees ambulance providers. Costs and expected medicare margins vary greatly, May 2007 www.gao.gov/new.items/d07383.pdf 